\title{
North American Medical Informatics (NAMI)
}

\author{
Andre Kushniruk ${ }^{1,2}$, Karen Greenwood ${ }^{3}$, Mark Casselman ${ }^{2}$ \\ School of Health Information Science, University of Victoria, Victoria, Canada \\ 2 Digital Health Canada, Toronto, Ontario, Canada \\ ${ }^{3}$ American Medical Informatics Association, Bethesda, MD, USA
}

This article reports on North America for both the United States (AMIA) and Canada (Digital Health Canada). In both countries there have been significant moves to broaden the scope of the national informatics assocations and increase the range and scope of memberships. Both countries continue to expand their programs, events, and work in areas such as education, certification, and exposure and dissemination of advances at all levels of the healthcare ecosystem.

\section{Informatics Activities in the United States - Report from AMIA}

AMIA - Informatics Professionals. Leading the Way.

AMIA represents more than 5,600 healthcare professionals, students, informatics researchers, practitioners, and thought-leaders in biomedicine, healthcare, and science. AMIA's members are subject matter experts in the science and practice of informatics as it relates to clinical care, research, education, and policy. They address challenges across the continuum of the health ecosystem-consumers and patients, healthcare providers and care delivery systems, population and public health, and basic and clinical research with the ultimate goal to advance better health, better healthcare, and improved efficiency through the use of informatics and information technology.

\section{Why Informatics?}

In 2019, AMIA will continue its communication campaign - promoting the fact that every day people benefit from infor- maticians' ability to accelerate healthcare's transformation by collecting, analyzing and applying data directly to care decisions. The campaign features AMIA members telling their stories on video. Members share how informatics is connected to real-world outcomes by lowering costs, increasing safety and promoting the use of high-quality services.

For more information and to share the videos with colleagues and students, visit https:// www.amia.org/why-informatics.

\section{Meetings and Conferences}

AMIA meetings offer education, networking, and peer-reviewed presentation opportunities reflecting the comprehensive range of informatics domains serving hundreds of health and healthcare related disciplines. AMIA meetings are widely regarded as the world's most comprehensive meetings on clinical and health informatics and provide a broad range of sessions on timely topics of informatics.

\section{AMIA 2019 Informatics Summit}

Bioinformatics | Clinical Research | Implementation $\mid$ Data Science

March 25 - 28, San Francisco, CA

The Informatics Summit follows the tradition of offering an exciting, innovative and engaging program around four main areas: Translational Bioinformatics, Clinical Research Informatics, Implementation Informatics, and Data Science. The program serves clinical and translational investigators, health data scientists, computational biologists, genomics researchers, public health geneticists, clinical and public health informaticians, and policy professionals focused on the research enterprise.

AMIA 2019 Clinical Informatics Conference Evidence | Analytics | Best Practice April 30 - May 2, Atlanta GA

At the Clinical Informatics Conference clinicians, data scientists, HIT developers and researchers gather to learn about implementing team-based, integrated healthcare driven by data, evidence, and best practice. This is the "doing conference" where workshops and presentations provide attendees with tools to help them in their day-to-day practice. At this venue evidence and experience-based clinical informatics are explored to improve the quality and safety of health care. We are particularly excited to bring the AMIA 2019 Clinical Informatics Conference (CIC) to Atlanta as we strive to facilitate collaboration between the clinical and public health informatics communities.

AMIA 2019 Informatics Educators Forum Scholarship | Leadership | Professional Advancement

June 18 - 20, St. Louis, MO

The Informatics Educators Forum provides an opportunity to learn state-of-the-art approaches and best practices in education, research, and training; and to build, support, and strengthen the academic informatics community. The conference is designed for faculty and staff who are committed to their own professional growth and the growth of their program. The Informatics Educators Forum also serves multidisciplinary, inter-professional informatics professionals at all levels of academic informatics. 
AMIA 2019 Annual Symposium November 16 - 20, Washington, DC

AMIA's flagship annual meeting draws the multidisciplinary, interprofessional community of informaticians together for five intensive days of learning and networking. It features hundreds of scientific sessions, workshops, exhibition, posters, and opportunities displaying the full breadth and depth of the informatics profession.

\section{Publications}

\section{JAMIA}

JAMIA continues to provide members and subscribers with the best in biomedical and health informatics publishing as the premier journal in the field. Suzanne Bakken was named as the new editor-inchief beginning in 2019. She and her team will focus on continuing the tradition of excellence in informatics publishing with a specific focus on providing a consequentialist approach. Her first editorial shares her thoughts https://academic.oup.com/jamia/ article/26/1/1/5260826

\section{JAMIA Open}

JAMIA Open, AMIA's peer-reviewed, online-only, and gold open access journal continues to be led by Neil Sarkar. JAMIA Open provides a global forum for the publication of novel research and insights in the major areas of informatics for biomedicine and health (e.g., translational bioinformatics, clinical research informatics, clinical informatics, public health informatics, and consumer health informatics), as well as related areas such as data science, qualitative research, and implementation science. In 2019, the journal will feature a special issue focused on precision medicine in the patient-centered era.

\section{ACI (Applied Clinical Informatics)}

AMIA members continue to receive the ACI journal as part of their membership. As the official eJournal of AMIA and IMIA led by Christoph Lehmann, ACI aims to establish a platform that allows sharing knowledge between clinical medicine and health IT specialists as well as bridging gaps between visionary design and successful and pragmatic deployment.

\section{ACI Open}

ACI Open is the open access companion journal to ACI led by David Dorr and Lipika Samal. The journal publishes subject matters in the field of clinical informatics including clinical information systems, mobile health applications, and administrative and management systems.

\section{Certifications and Accreditation Activities}

The field of clinical and health informatics is changing in the United States. What was once only an interdisciplinary field of research is now an active area of research and a growing profession. The American Board of Medical Specialties (ABMS) established the medical subspecialty of clinical informatics in 2011 and now there are over 1,700 board-certified clinical informatics professionals in the US. There has been similar growth in health informatics in other health professions. While research remains an important part of informatics activities, more and more AMIA members identify as clinical and health informatics professionals in applied positions.

\section{Clinical Informatics Practice Analysis}

AMIA has a longstanding commitment to supporting the Clinical Informatics Subspecialty (CIS). AMIA conducted the work that served as the foundation for the clinical informatics subspecialty between 2007 and 2009 - including development of the CIS Core Content. The practice of clinical informatics has changed considerably since that work was completed. During the past decade, the practice of clinical informatics has evolved in response to:

- Significant advances in health care delivery, information technology, and electronic health records (EHRs)

- Deeper understanding of how health information technology impacts clinical workflow, clinician productivity and satisfaction, and patient experiences

- Increased focus on using data from EHRs to support research, precision medicine, and population health management (huge health data stores, much of which continues to be "locked" in text)

- Scientific advances in -omics, including phenomics, drive integration of new data types and analyses in health care
- Changes outside healthcare and HIT, such as ever-higher user expectations on how they interact with computational resources, are influencing the knowledge and skills needed by CIS professionals.

In response to these rapid changes, and to keep our understanding of the clinical informatics subspecialty current, we have completed a comprehensive update to the skills and competencies that underlie the CIS. The results of this study will be used to update the CIS examination, and as the foundation to update the Accreditation Council for Graduate Medical Education (ACGME) accreditation standards for clinical informatics fellowships.

\section{Health Informatics Practice Analysis}

AMIA has always been committed to supporting opportunities for professional recognition for the full spectrum of professionals, who practice health informatics. AMIA proposed advanced health informatics certification (AHIC) eligibility requirements in 2016. In 2017, AMIA completed Core Competencies for Health Informatics Education at the Master's Degree Level, which the Commission on Accreditation for Health Informatics and Health Information Management Education (CAHIIM) incorporated into its accreditation requirements.

In 2018, AMIA continued to explore how to establish AHIC and credentials that will meet the needs of informatics professionals earlier in their careers. To advance work on this front, AMIA initiated a formal practice analysis for health informatics professionals that will be completed in 2019. The results of this practice analysis will:

- For the first time, describe the practice of health informatics in terms of competencies and knowledge

- Inform eligibility requirements, test specifications, and maintenance of certification for future $\mathrm{HI}$ certifications

- Inform a career pathway for health informatics

The results of both the Clinical Informatics Subspecialty and the health informatics practice analysis will be published in JAMIA in 2019. We look forward to sharing this information with our international colleagues. 
Certification and Accreditation References

- Detmer DE, Lumpkin JR, Williamson JJ, Defining the medical subspecialty of clinical informatics. J Am Med Inform Assoc. 2009;16:167-168.

- Gadd CG, Williams JJ, Steen EB, Fridsma DF. Creating advanced health informatics certification. J Am Med Inform Assoc 2016;4:848-850.

- Gadd CG, Williams JJ, Steen EB, et al. Eligibility requirements for advanced health informatics certification. J Am Med Inform Assoc 2016;4:851-854.

- Gardner RM, Overhage JM, Steen EB, et al. Core content for the subspecialty of clinical informatics. J Am Med Inform Assoc 2009;2:153-157.

- Lehmann CU, Gundlapalli AV, Williamson JJ, et al. Five years of clinical informatics board certification for physicians in the United States of America. IMIA Yearbook of Medical Informatics 2018:237-242.

- Safran C, Shabot MM, Munger BS, et al. Program requirements for fellowship education in the subspecialty of clinical informatics. J Am Med Inform Assoc 2009;2:158-166. Erratum in: J Am Med Inform Assoc 2009;4:605.

- Valenta AL, Berner ES, Boren SA et al. AMIA Board White Paper: AMIA 2017 core competencies for applied health informatics education at the master's degree level. . J Am Med Inform Assoc 2018; 12: $1657-1668$.

\section{For more information}

AMIA

4720 Montgomery Lane, Suite 500

Bethesda, MD 20814

Tel: 3016571291

Fax: 3016571296

Email: mail@amia.org

Website: www.amia.org

Twitter: @AMIAinformatics

LinkedIn: Official Group of AMIA

Facebook: American Medical Informatics Association

\section{Informatics Activities in Canada - Report from Digital Health Canada}

\section{Current Membership and Direction}

Digital Health Canada has a broad membership with over 2,500 members, including clinicians, innovators, students, researchers, thought leaders, and practitioners at all levels. The association continues to maintain a global and holistic perspective on the field of health informatics and digital health and works to connect, inspire, and educate health professionals who are shaping the future of health in Canada. The organization aims to catalyze the digital health community, develop knowledge, and demonstrate leadership in health informatics through supporting collaboration and sharing of health informatics and digital health knowledge and experience. Digital Health Canada is committed to continuous learning, while being guided by professionalism and ethics, and responding to the emerging needs of its members and the health system.

\section{Conferences and Events - 2018}

eHealth 2018: Celebrate, Grow \& Inspire Bold Action in Digital Health, was held in Vancouver from May 27-30, 2018 and focused on the past, current, and future state of Canada's digital health community. The conference had over 1,500 attendees participating in more than 250 educational sessions and over 85 exhibitors, a "Start Up Zone" for new companies, and the Hacking Health@e-Health 2018 event. The Canadian Health Informatics (CHIA) Gala at e-Health celebrated thought leaders, innovative industry leaders, and emerging health informatics professionals. A well-attended pre-conference symposium focused on patient engagement, with clinicians and patient representatives sharing insights and experiences.

Webinar Wednesdays continue to provide a convenient online way for health professionals to keep up with emerging issues, trends, and ideas in health informatics and digital health. The weekly webinar topics have varied, including telehealth, standards, privacy and security, data quality, patient centered care, consumer health informatics, and other timely and important topics aimed to keep attendees up to date.

6th Annual Calgary Winter Digital Health Canada/ANHIX Conference - This event was held on February 6, 2018 and explored effective care delivery issues and opportunities and examined current initiatives and technology innovations. Attendees discussed moving healthcare delivery outside the walls of traditional hospitals and institutions and facilitating patient involvement.

\section{2nd Annual 'Ahead of the Curve' Conference} took place in Vancouver on February 8, 2018. Thought leaders shared perspectives and led discussion on engaging patients in their care with emerging technologies and innovative care models and developing new solutions. Attendees discussed solutions from machine learning and artificial intelligence and incorporating connected personal health services, new devices, and increasing data to reimagine healthcare.

UPONDIGITAL: The Update on Ontario Digital Health took place in Toronto on March 26, 2018 and featured speakers discussing the innovation agenda from the public sector and government perspective. Challenges and opportunities in innovation in Ontario were discussed. The program focused on exploring innovation in procurement and featured presentations and discussions about how new approaches to procurement can enable the spread and increase the scale of digital solutions. Public and private sector leaders from across the province participated in the discussions.

Canada's Health Informatics Executive Forum (CHIEF) Spring Symposium 2018 took place in Vancouver on May 25, 2018. CHIEFs from across Canada met to build trusted relationships, collaborate on finding solutions for the issues facing healthcare today, and discuss the future of digital health in Canada.

The CHIEF Fall Symposium took place in Toronto on October 19 and 20, 2018. Topics covered over two days included Canada's Economic Strategy Tables; Enterprise Innovation; Population Health and Precision Medicine; and Data Interoperability. 
Attendance at the 2nd Annual Driving the Future of Digital Health Conference was twice as high as the previous year, with over 200 Ontario digital health professionals converging in Toronto to discuss the future of healthcare in a digital world, virtual care, bots and robotics, and the end-user in digital health.

\section{Programs, Services and Resources}

Digital Health Canada continues to offer a wide array of programs, services, and resources, including individual, academic, entrepreneur, and institutional membership levels. CHIEF (Canada's Health Informatics Executives Forum) continues to provide a channel for thought leaders to meet and share expert-level knowledge.

Communities of Action (CoA) offer members opportunities for connection, idea sharing, and professional development. Community of Action Working Groups come together to enhance the quality of healthcare for Canadians by learning more about an important digital health topic, meeting virtually to learn, collaborate, and share knowledge with the Canadian digital health community through reports, white papers, guidelines, or presentations.

Digital Health Canada also offers professional education in the form of Online, OnDemand, and Custom Health Informatics courses that provide a deep understanding of the Canadian healthcare system and the critical topics underlying health informatics. Digital Health Canada education prepares professionals to achieve the Certified Professional in Healthcare Information and Management Systems - Canada (CPHIMSCA) credential.

The association continues to work closely with the academic programs at universities and colleges across Canada. Influential work includes the CPHIMS-CA Certification, the HIP ${ }^{\circledR}$ Competency framework, HIP ${ }^{\circledR}$ core competencies (which have been used to guide curricula in health informatics throughout Canada and internationally), HIP ${ }^{\circledR}$ career matrix, $H I P \AA$ role profiles and the Interactive HIP ${ }^{\circledR}$ Career Matrix.

Digital Health Canada announced the formation of a new chapter in Atlantic Canada with a goal of connecting members in Newfoundland, Nova Scotia, Prince Edward Island, and New Brunswick. The establishment of the first regional chapter, to be known as the Atlantic Chapter, aligns with the association's mission to connect, inspire, and educate the digital health professionals creating the future of health in Canada.

In December 2018, Digital Health Canada announced their partnership with Hacking Health, a non-for-profit organization with 600 volunteers working in 53 chapters around the world. The partnership will enable cross-sector stakeholders to form new connections and strengthen existing relationships as they work to create the future of health in Canada.

\section{Publications}

- Journal: Healthcare Information Management and Communications Canada (January 2018/March 2018/May 2018)

- Report: A Before B - Perspectives on Artificial Intelligence in Healthcare

- Case Study: More than a Registry: Diabetes Care Program of Nova Scotia

- Report: e-Safety Update 2017-2018

- Whitepaper: Canada and Shared Information Governance

\section{Upcoming Events for 2019}

Advancing Healthcare Through Technology Digital Health Canada/ANHIX Calgary Winter Conference

February 5, 2019 | Calgary, Alberta

Featuring a keynote on unlocking the full potential of innovation in healthcare and panels on topics such as artificial intelligence and machine learning for health, virtual reality in wound care, and bridging technology and the clinical setting.

\section{Ahead of the Curve}

Digital Health Canada Vancouver Conference February 7, 2019 | Vancouver, BC

Offering a first look at British Columbia's Digital Health Transformation Imperative plus panels focusing on Virtual Care, Patient Engagement, Mental Health Technology Resources, and Canada's Digital Technology Super Cluster.

\section{UpOnDigital}

The Update on Ontario Digital Health March 4, 2019 | Toronto, Ontario

Giving public sector leaders a forum to provide a state-of-the-union-type address with special selected themes relevant to digital health.

e-Health Conference and Tradeshow 2019 May 26-29, 2019 | Toronto, Ontario

Canada's only national digital health event brings together more than 1500 digital health professionals annually.

Digital Health Canada continues to focus on growth, expanded opportunities, new partnerships, enhanced networking, and creating and sponsoring a range of events and opportunities to facilitate the effective spread of digital health in Canada.

\section{For more information:}

Digital Health Canada 11th Floor, 151 Yonge Street Toronto, Ontario M5C 2W7 Canada digitalhealthcanada.com Twitter | LinkedIn | Instagram | Facebook 